Tôhoku Math. Journ.

25 (1973), 461-467.

\title{
A PINCHING PROBLEM ON THE SECOND FUNDAMENTAL TENSORS AND SUBMANIFOLDS OF A SPHERE
}

\author{
Dedicated to Professor Shigeo Sasaki on his 60th birthday \\ MASAFUMi OKUMURA
}

(Received November 28, 1972; Revised March 12, 1973)

Introduction. In a previous paper [4] the present author proved the following

THEOREM A. Let $M$ be an n-dimensional compact, connected hypersurface with constant mean curvature immersed in an $(n+1)$-dimensional Riemannian manifold of non-negative constant curvature. If the second fundamental tensor $H$ satisfies

$$
\text { trace } H^{2}<\frac{1}{n-1}(\operatorname{trace} H)^{2},
$$

then $M$ is a totally umbilical hypersurface and consequently a sphere.

Then in [5] we generalized Theorem A to a submanifold of any codimension and proved.

THEOREM B. Let $M$ be a compact, connected submanifold of dimension $n$ immersed in an $(n+p)$-dimensional Riemannian manifold of nonnegative constant curvature and suppose that the connection of the normal bundle is flat. If the mean curvature vector field is parallel with respect to the connection of the normal bundle and the inequality

$$
\sum_{A=1}^{p} \operatorname{trace} H_{A}^{2}<\frac{1}{n-1} \sum_{A=1}^{p}\left(\operatorname{trace} H_{A}\right)^{2}
$$

is satisfied, then $M$ is a totally umbilical submanifold, where $H_{A}$ 's are the second fundamental tensors with respect to unit normals $N_{A}$.

The purpose of the present paper is to prove the following

THEOREM. Let $M$ be a complete, connected submanifold of dimension $n(\geqq 3)$ immersed in an $(n+p)$-dimensional Riemannian manifold of positive constant curvature whose mean curvature vector field is parallel with respect to the connection of the normal bundle. If the second fundamental tensors $H_{A}$ satisfy $(0.2)$, then $M$ is umbilical with respect to the 
mean curvature normal direction. Furthermore, if the ambient manifold is an $(n+p)$-dimensional sphere $(n \geqq 3), M$ is a minimal submanifold of a small sphere.

1. Preliminaries. Let $M$ be an $n$-dimensional submanifold of an $(n+p)$-dimensional Riemannian manifold $\bar{M}$ of constant curvature $c$. The Riemannian connections of $M$ and $\bar{M}$ are denoted by $\nabla$ and $\bar{\nabla}$ respectively, whereas the connection in the normal bundle of $M$ in $\bar{M}$ is denoted by $D$. Let $N_{1}, \cdots, N_{p}$ be mutually orthogonal unit normal vectors at a point $p \in M$ and extend them to local vector fields in a neighborhood of $p$. We define $-H_{A} X(A=1,2, \cdots, p)$ to be the tangential components of $\bar{V}_{X} N_{A}$ for $X \in T_{p}(M)$ and call $H_{A}$ the second fundamental tensor with respect to $N_{A}$. We know that the $H_{A}$ 's are symmetric linear transformations on $T_{p}(M)$. Then we have the following equations of Gauss and Weingarten:

$$
\begin{gathered}
\bar{\nabla}_{X} Y=\nabla_{X} Y+\sum_{A=1}^{p} g\left(H_{A} X, Y\right) N_{A}, \\
\bar{\nabla}_{X} N_{A}=-H_{A} X+D_{X} N_{A},
\end{gathered}
$$

where $g$ is the Riemannian metric of $M$. Since $D_{X} N_{A}$ is normal to $M$, it is expressed as a linear combination of $N_{A}$, that is,

$$
D_{X} N_{A}=\sum_{B=1}^{p} S_{A B}(X) N_{B} .
$$

The ambient manifold being of constant curvature $c$, the curvature tensor $R(X, Y) Z$, scalar curvature $K$, and the normal curvature $R^{N}$ are respectively given by

$$
\begin{aligned}
& R(X, Y) Z= c\{g(Y, Z) X-g(X, Z) Y\} \\
&+\sum_{A=1}^{p}\left\{g\left(H_{A} Y, Z\right) H_{A} X-g\left(H_{A} X, Z\right) H_{A} Y\right\}, \\
& K=n(n-1) c+\sum_{A=1}^{p}\left(\operatorname{trace} H_{A}\right)^{2}-\sum_{A=1}^{p} \operatorname{trace} H_{A}^{2}, \\
& R^{N}(X, Y) N_{A}= \sum_{B=1}^{p} g\left(\left[H_{A}, H_{B}\right] X, Y\right) N_{B} \\
&= \sum_{B=1}^{p}\left\{\left(\nabla_{X} S_{A B}\right) Y-\left(\nabla_{Y} S_{A B}\right) X\right. \\
&\left.+\sum_{C=1}^{p}\left(S_{A C}(Y) S_{C B}(X)-S_{A C}(X) S_{C B}(Y)\right)\right\} N_{B},
\end{aligned}
$$

where we put

$$
\left[H_{A}, H_{B}\right] X=H_{A} H_{B} X-H_{B} H_{A} X .
$$


The mean curvature vector $N$ is defined by

$$
N=\sum_{A=1}^{p}\left(\operatorname{trace} H_{A}\right) N_{A},
$$

and it is well known that $N$ is independent of the choice of unit normal vector to $M$.

For some $H_{A}$, if there exists a function $\rho_{A}$ such that

$$
H_{A} X=\rho_{A} X,
$$

at each point of $M$, we call $M$ is umbilical with respect to normal $N_{A}$ at $p$.

2. Lemmas. First we state the following

Lemma 1. [5]. Let $a_{1}, a_{2}, \cdots, a_{n}$ and $k$ be $n+1(n \geqq 2)$ real numbers satisfying the inequality

$$
\sum_{i=1}^{n} a_{i}^{2}+k<\frac{1}{n-1}\left(\sum_{i=1}^{n} a_{i}\right)^{2},
$$

then for any pair of distinct $i$ and $j=1,2, \cdots, n$, we have

$$
k<2 a_{i} a_{j} \text {. }
$$

Proof. Since $\left(\sum_{i=1}^{n} a_{i}\right)^{2}=\sum_{i=1}^{n} a_{i}^{2}+2 \sum_{i<j}^{n} a_{i} a_{j}$, we have from (2.1),

$$
(n-2) \sum_{i=1}^{n} a_{i}^{2}-2 \sum_{i<j}^{n} a_{i} a_{j}+(n-1) k<0,
$$

that is,

$$
(n-2) a_{n}^{2}-2 a_{n}\left(\sum_{i=1}^{n-1} a_{i}\right)+(n-2) \sum_{i=1}^{n-1} a_{i}^{2}-2 \sum_{i<j \leq n-1} a_{i} a_{j}+(n-1) k<0 .
$$

We regard that (2.3) is a quadratic inequality with respect to $a_{n}$. Then, $a_{n}$ being a real number, the discriminant of (2.3) must be positive. Thus we get

$$
\left(\sum_{i=1}^{n-1} a_{i}\right)^{2}>(n-2)\left\{(n-1)\left(\sum_{i=1}^{n-1} a_{i}^{2}+k\right)-\left(\sum_{i=1}^{n-1} a_{i}\right)^{2}\right\},
$$

from which

$$
\sum_{i=1}^{n-1} a_{i}^{2}+k<\frac{1}{n-2}\left(\sum_{i=1}^{n-1} a_{i}\right)^{2} .
$$

Continuing the same process $(n-2)$-times, we have (2.2).

Next we prove the

LEMMA 2. Let $M$ be an n-dimensional submanifold of a Riemannian manifold $M$ of constant curvature $c$. If the second fundamental tensors 
$H_{A}$ satisfy (0.2) at a point $p \in M$, then the sectional curvature $R(i, j)$ for the plane section spanned by $E_{i}$ and $E_{j}$ is greater than $c$ at $p$.

Proof. From (0.2) it follows that $M$ has no minimal point. So we can choose the first unit normal vector $N_{1}$ to $M$ in the direction of the mean curvature vector $N$. Then by the definition of the mean curvature vector we can easily see that

$$
\text { trace } H_{A}=0, \quad A=2,3, \cdots, p .
$$

Let $E_{1}, E_{2}, \cdots, E_{n}$ be orthonormal eigenvectors of the second fundamental tensor $H_{1}$ and $a_{1}, a_{2}, \cdots, a_{n}$ corresponding eigenvalues to $E_{1}, E_{2}, \ldots$, $E_{n}$. Then denoting components of $H_{A}(A=2, \cdots, p)$ by $\lambda_{j i}^{A}$, we have, from (0.2) and (2.5),

$$
\frac{1}{n-1}\left(\sum_{i=1}^{n} a_{i}\right)^{2}>\sum_{i=1}^{n} a_{i}^{2}+\sum_{A=2}^{p} \sum_{i, k=1}^{n}\left(\lambda_{i k}^{A} \lambda_{i k}^{A}\right) .
$$

Applying Lemma 1 to (2.6), we have

$$
2 a_{i} a_{j}>\sum_{A=2}^{p} \sum_{i, k=1}^{n}\left(\lambda_{i k}^{A} \lambda_{i k}^{A}\right) \geqq \sum_{A=2}^{p}\left\{\left(\lambda_{i i}^{A}\right)^{2}+2\left(\lambda_{i j}^{A}\right)^{2}+\left(\lambda_{j j}^{A}\right)^{2}\right\} \geqq 2 \sum_{A=2}^{p}\left\{\left|\lambda_{i i}^{A} \lambda_{j j}^{A}\right|+\left(\lambda_{i j}^{A}\right)^{2}\right\} .
$$

Thus we have

$$
a_{i} a_{j}>\sum_{A=2}^{p}\left\{\left|\lambda_{i i}^{A} \lambda_{j j}^{A}\right|+\left(\lambda_{i j}^{A}\right)^{2}\right\} .
$$

On the other hand, by (1.4), the sectional curvature $R(i, j)$ for the plane section spanned by $E_{i}$ and $E_{j}$ is given by

$$
R(i, j)=g\left(R\left(E_{i}, E_{j}\right) E_{j}, E_{i}\right)=c+a_{i} a_{j}+\sum_{A=2}^{p}\left\{\lambda_{i i}^{A} \lambda_{j j}^{A}-\left(\lambda_{i j}^{A}\right)^{2}\right\} .
$$

Combining (2.7) and (2.8) we have

$$
R(i, j)>c+\sum_{A=2}^{p}\left\{\left|\lambda_{i i}^{A} \lambda_{j j}^{A}\right|+\lambda_{i i}^{A} \lambda_{j j}^{A}\right\} \geqq c .
$$

This completes the proof.

LEMMA 3. Let $M$ be a complete, connected submanifold of dimension $n>2$ immersed in an $(n+p)$-dimensional Riemannian manifold of positive constant curvature $c$. If the second fundamental tensors $H_{A}$ satisfy $(0.2)$ on $M$, then $M$ is compact.

PRoof. Let $X$ be a unit vector and $E_{i}$ be a unit eigenvector of $H_{1}$ which corresponds to the eigenvalue $a_{i}$. Then, putting $X=\sum_{j=1}^{n} x^{j} E_{j}$, the sectional curvature for the plane section spanned by $X$ and $E_{i}$ is 


$$
\begin{aligned}
g\left(R\left(X, E_{i}\right) E_{i}, X\right)= & c\left\{\sum_{j=1}^{n}\left(x^{j}\right)^{2}-\left(x^{i}\right)^{2}\right\}+a_{i} \sum_{j=1}^{n} a_{j}\left(x^{j}\right)^{2}-\left(a_{i} x^{i}\right)^{2} \\
& +\sum_{A=2}^{p}\left\{\lambda_{i i}^{A} \sum_{j, k=1}^{n} \lambda_{j k}^{A} x^{j} x^{k}-\left(\sum_{j=1}^{n} \lambda_{j i}^{A} x^{j}\right)^{2}\right\}
\end{aligned}
$$

Thus Ricci tensor $\operatorname{Ric}(X, X)$ becomes

$$
\begin{aligned}
\operatorname{Ric}(X, X)= & \sum_{i=1}^{n} g\left(R\left(X, E_{i}\right) E_{i}, X\right) \\
= & (n-1) c+\sum_{i=1}^{n}\left\{a_{i} a_{1}\left(x^{1}\right)^{2}+\cdots+\widehat{a_{i}{ }^{2}\left(x^{i}\right)^{2}}+\cdots+a_{i} a_{n}\left(x^{n}\right)^{2}\right\} \\
& -\sum_{A=2}^{p} \sum_{i=1}^{n}\left(\sum_{j=1}^{n} \lambda_{j i}^{A} x^{j}\right)^{2},
\end{aligned}
$$

because of $\sum_{i=1}^{n} \lambda_{i i}^{A}=0$, where the roof " $\wedge$ " denotes a term which will be omitted.

Substituting

$$
2 a_{i} a_{h}>\sum_{A=2}^{p} \sum_{j, k=1}^{n} \lambda_{j k}^{A} \lambda_{j k}^{A}
$$

into the last equation and making use of $\sum_{i=1}^{n}\left(x^{i}\right)^{2}=1$, we have

$$
\begin{aligned}
& \operatorname{Ric}(X, X)>(n-1) c+\sum_{A=2}^{p}\left\{\frac{n-1}{2} \sum_{j, k=1}^{n} \lambda_{j k}^{A} \lambda_{j k}^{A}-\sum_{i=1}^{n}\left(\sum_{j=1}^{n} \lambda_{j i}^{A} x^{j}\right)^{2}\right\} \\
& \geqq(n-1) c+\sum_{A=2}^{p}\left\{\frac{n-1}{2} \sum_{j, k=1}^{n} \lambda_{j k}^{A} \lambda_{j k}^{A}-\sum_{i=1}^{n}\left(\lambda_{j i}^{A} \lambda_{j i}^{A}\right) \sum_{k=1}^{n}\left(x^{k}\right)^{2}\right\} \\
& =(n-1) c+\frac{n-3}{2} \sum_{A=2}^{p} \sum_{j, k=1}^{n} \lambda_{j k}^{A} \lambda_{j k}^{A} \geqq(n-1) c>0,
\end{aligned}
$$

where we have used Cauchy-Schwarz inequality. Thus, from Myers' theorem [3], $M$ is compact.

3. Proof of Theorem. Let $f$ be the square of the length of the second fundamental tensor with respect to $N_{1}$, that is,

$$
f=\operatorname{trace} H_{1}^{2} \text {. }
$$

The Laplacian for $f$ is given by

$$
\frac{1}{2} \Delta f=\operatorname{trace}\left(\Delta^{\prime} H_{1}\right) H_{1}+g\left(\nabla H_{1}, \nabla H_{1}\right) \text {, }
$$

where

$$
\left(\Delta^{\prime} H_{1}\right)_{X}=\sum_{i=1}^{n}\left\{\nabla_{E_{\boldsymbol{i}}}\left(\nabla_{E_{\boldsymbol{i}}} H_{1}\right)-\nabla_{\nabla_{E_{\boldsymbol{i}}} E_{\boldsymbol{i}}} H_{1}\right\},
$$

and we extend the metric $g$ to the tensor space in the standard fashion. 
Using recent results of J. A. Erbacher [2], we have

$$
\begin{aligned}
\Delta^{\prime} H_{1}= & n c H_{1}-c\left(\operatorname{trace} H_{1}\right) I+\sum_{A=1}^{p}\left(\operatorname{trace} H_{A}\right) H_{1} H_{A} \\
& -\sum_{A=1}^{p}\left(\operatorname{trace} H_{A} H_{1}\right) H_{A}+\sum_{A=1}^{p}\left[H_{A}, H_{1} H_{A}\right]+\sum_{A=1}^{p} H_{A}\left[H_{1}, H_{A}\right] \\
& +\sum_{i=1}^{n} \sum_{A=1}^{p}\left(\nabla_{E_{i}} S_{1 A}\right)\left(E_{i}\right) H_{A}+2 \sum_{i=1}^{n} \sum_{A=1}^{p} S_{1 A}\left(E_{i}\right) V_{E_{i}} H_{A} \\
& -\sum_{i=1}^{n} \sum_{A, B=1}^{p} S_{1 A}\left(E_{i}\right) S_{A B}\left(E_{i}\right) H_{B} .
\end{aligned}
$$

By the assumption of Theorem, $N_{1}$ is parallel with respect to the connection of the normal bundle and so we have

$$
D_{X} N_{1}=\sum_{A=1}^{p} S_{1 A}(X) N_{A}=0,
$$

from which $S_{1 A}=0$. Consequently we get $\left[H_{1}, H_{A}\right]=0$.

Substituting these into (3.3) and making use of (3.2), we have

$$
\begin{aligned}
\frac{1}{2} \Delta \operatorname{trace} H_{1}^{2}= & n c \operatorname{trace} H_{1}^{2}-c\left(\operatorname{trace} H_{1}\right)^{2}+\left(\operatorname{trace} H_{1}\right)\left(\operatorname{trace} H_{1}^{3}\right) \\
& +\sum_{A=2}^{p} \operatorname{trace}\left(H_{A} H_{1}\right)^{2}-\left(\operatorname{trace} H_{1}^{2}\right)^{2}-\sum_{A=2}^{p}\left(\operatorname{trace} H_{A}^{2} H_{1}^{2}\right) \\
& -\sum_{A=2}^{p}\left(\operatorname{trace} H_{A} H_{1}\right)^{2}+g\left(\nabla H_{1}, \nabla H_{1}\right) .
\end{aligned}
$$

Thus at a point $p \in M$, we have

$$
\begin{aligned}
& \frac{1}{2} \Delta \text { trace } H_{1}^{2}=n c\left(\sum_{i=1}^{n} a_{i}^{2}-\frac{1}{n}\left(\sum_{i=1}^{n} a_{i}\right)^{2}\right) \\
& \quad+\sum_{i<j}\left(a_{i} a_{j}+\sum_{A=2}^{n}\left(\lambda_{i i}^{A} \lambda_{j j}^{A}-\left(\lambda_{i j}^{A}\right)^{2}\right)\right)\left(a_{i}-a_{j}\right)^{2}+g\left(\nabla H_{1}, \nabla H_{1}\right) \\
& \geqq n c\left(\sum_{i=1}^{n} a_{i}^{2}-\frac{1}{n}\left(\sum_{i=1}^{n} a_{i}\right)^{2}\right)+(R(i, j)-c)\left(a_{i}-a_{j}\right)^{2}+g\left(\nabla H_{1}, \nabla H_{1}\right),
\end{aligned}
$$

because of (2.7), (2.8) and the fact that $\sum_{j>i} a_{i}^{2} \lambda_{i i}^{A} \lambda_{i j}^{A}=0$ for $A \geqq 2$.

From Lemma 3, $M$ is compact and so by Hopf's theorem and Lemma 2 , we see that $\nabla H_{1}=0$ and

$$
a_{1}=a_{2}=\cdots=a_{n} \neq 0 .
$$

This shows that $M$ is umbilical with respect to the mean curvature vector $H_{1}$ and $H_{1}$ is parallel. Furthermore, if the ambient manifold $\bar{M}$ is an $(n+p)$-dimensional sphere in Euclidean $n+p+1$ space $E^{n+p+1}$, then, using $D_{X} H_{1}=0$, we have, for example by [1] or [7], that $M$ is a minimal submanifold of a sphere $S^{n+p-1}$. This completes the proof. 
Remark. From (1.5), the condition (0.2) can be written as

$$
K>n(n-1) c+(n-2) \sum_{A=1}^{n} \operatorname{trace} H_{A}^{2} .
$$

\section{BIBLIOGRAPHY}

[1] B. Y. Chen And K. Yano, Pseudo-umbilical submanifolds in a Riemannian manifold of constant curvature, Differential Geometry, in honor of K. Yano, Kinokuniya Tokyo, 1972, 61-71.

[2] J. ERBACHER, Isometric immersions of constant mean curvature and triviality of the normal connection, Nagoya Math. J., 45 (1971), 139-165.

[3] S. Myers, Riemannian manifolds with positive mean curvature, Duke Math. J., 8(1941), 401-404.

[4] M. OKUMURA, Hypersurfaces and a pinching problem on the second fundamental tensor, to appear.

[5] M. OKUMURA, Submanifolds and a pinching problem on the second fundamental tensor, Transactions of A.M.S. 178 (1973), 285-291.

[6] J. Simons, Minimal varieties in riemannian manifolds, Ann. of Math., 88(1968), 62-105.

[7] B. Sмчтн, Submanifolds of constant mean curvature, Mathematics Annalen 205 (1973), 265-280.

Michigan State University

AND

SAITAMA UNIVERSITY 
